\title{
1. A Model-Based Framework for Fault Estimation and 2 Accommodation Applied to Distributed Energy \\ 3
}

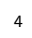

6

8
James T. Allen and Nael H. El-Farra ${ }^{\mathrm{a}}$

Department of Chemical Engineering \&s Material Science, University of California, Davis, CA 95616 (e-mail: nhelfarra@ucdavis.edu).

\begin{abstract}
This paper presents the development and approach of a model-based fault identification and accommodation framework applied to sampled-data controlled distributed energy resources subject to control actuator faults. The main objective of the proposed approach is to handle faults that degrade stability as well as performance, while remaining robust to false alarms. The proposed method allows for dual fault detection and estimation, through the use of an embedded system model that minimizes the residual between the estimated and sampled states at each sampling period by adjusting a fault parameter in the embedded model over a past horizon. The resulting fault parameter estimate is then used by the control system to find an optimal fault accommodation strategy by minimizing a predefined performance metric whilst ensuring closed-loop stability. The developed fault accommodation framework is then applied to a simulated model of a solid oxide fuel cell subject to both stability and performance degrading faults in the control actuators. A discussion of some of the practical implementation issues associated with the developed framework is also included. Keywords: Fault estimation; Fault accommodation; Model-based control, Sampled-data systems, Distributed energy resources
\end{abstract}

\section{Introduction}

Distributed energy resources (DERs) are composed of modular energy generation units including, for example, micro-turbines, fuel cells, renewable energy

Preprint submitted to Renewable Energy Journal

April 29, 2016 
systems, battery storage and other such technologies deployed close to the point of consumption. The modular nature of DERs allows them to be integrated with existing grid infrastructure or implemented in a stand-alone manner. DERs offer advantages over conventional grid electricity by offering end users a diversified fuel supply; higher power reliability, quality, and efficiency; lower emissions and greater flexibility to respond to changing energy needs. While DERs have aided in the integration of sustainable energy resources into the power grid, they also pose fundamental challenges, including fluctuations in generation from intermittent availability of renewable resources as well as the resulting increased communication between generation and loads.

These and other challenges have been the subject of a significant and growing body of research work on the control of DERs (e.g., [1, 2, 3, 4, 5, 6]). Important contributions in this direction include the use of conventional and model-based feedback control algorithms to regulate various types of grid-connected DERs in order to enhance power system stability (e.g., 7, 8, 9, 10]), mitigate power quality problems (e.g., [11]) and improve the continuity of electricity supply (e.g., [12]), the development of various distributed control and coordination architectures using multi-agent systems (e.g., [13, 14]) and predictive control approaches (e.g., [15, 16]).

While control is necessary to ensure that the load demand is met and that economical operation of each DER is maintained, the stability and performance of smart grid DERs have not been rigorously assessed in the presence of faults or failures at the local and network levels. This is an important problem given the fact that the distributed power market is driven by the need for reliable highquality power, and the fact that local faults and disruptions in power flow can have a substantial impact, especially in situations when DERs are integrated to support grid operations. The timely identification and mitigation of faults at the local level before they cascade through the network are important capabilities that ensure autonomous control and protection, which when coupled with proper supervisory oversight enable distributed energy generation to provide highly reliable services under all disturbance and fault scenarios. In this context, fault- 
tolerant control is an important tool for reducing performance deterioration in the face of faults and uncertainties in the system components, such as actuators and sensors, resulting in increased reliability of the network.

In prior work $([17])$, fault-tolerant control has been studied in the context of a small-scale network of solid oxide fuel cells (SOFCs), where the focus has been on the detection and handling of destabilizing failure events at the local level without supervisory oversight. Local monitoring of the health status of each DER took place through use of a time-varying alarm threshold on a properly designed observer-based output residual. Exploiting the inherent actuator redundancy in SOFCs, three stabilizing controller configurations were designed, and a methodology for active switching between them in the event of a threshold breach was developed. The main contribution was the characterization of a stability region within which each controller configuration could operate. However, the faults considered in that work were limited to total failure events, thus negating the need for fault isolation or estimation. The proposed stability-based scheme also was not designed to detect faults that only degrade performance but do not compromise stability, and this can lead to sub-optimal performance.

Motivated by these considerations, this paper presents a framework for local actuator fault-tolerant control that accounts for both performance and stability degrading faults, while optimizing suitable performance metrics and maintaining closed-loop stability. The developed framework is realized through an integrated approach that brings together model-based control, data-based fault estimation and performance-based fault accommodation. The rest of the paper is organized as follows. A SOFC system is initially introduced in Section 2 to motivate the development and application of the proposed framework. This model system is used to design a local model-based state feedback controller that operates on sampled data and characterize its stability properties in the presence of faults. A fault estimation scheme that provides an estimate of the fault magnitude by solving a data-based moving horizon optimization problem is then introduced in Section 3, A performance index suitable for sampled-data systems is then introduced in Section 4 and used to develop a performance-based fault 
accommodation strategy that alters model and control parameters to minimize post-fault performance losses while maintaining closed-loop stability. Practical implementation issues, such as the need to minimize false alarms, are discussed and guidelines for dealing with these issues are presented. Finally, simulation results are presented in Section 5 .

\section{Preliminaries}

\subsection{Motivating example: A solid oxide fuel cell}

Due to their modular and stable nature SOFCs are of particular interest when it comes to decentralized distributed energy generation. A SOFC consists of two porous electrodes, an anode and a cathode, in contact with a solid metal oxide electrolyte between them. Hydrogen is fed along the surface of the anode where it releases electrons that migrate externally towards the cathode. The electrons combine with oxygen fed along the surface of the cathode to form oxide ions. These ions diffuse through the electrolyte towards the anode where they combine with the hydrogen ions to produce water and power. Under standard modeling assumptions (see [18]), the following dynamic model of the SOFC stack can be obtained from first principles: 


$$
\begin{aligned}
\dot{p}_{H_{2}}= & \frac{T_{s}}{\tau_{H_{2}}^{*} T^{*} K_{H_{2}}}\left(q_{H_{2}}^{i n}-K_{H_{2}} p_{H_{2}}-2 K_{r} I\right) \\
\dot{p}_{O_{2}}= & \frac{T_{s}}{\tau_{O_{2}}^{*} T^{*} K_{O_{2}}}\left(q_{O_{2}}^{i n}-K_{O_{2}} p_{O_{2}}-K_{r} I\right) \\
\dot{p}_{H_{2} O}= & \frac{T_{s}}{\tau_{H_{2} O}^{*} T^{*} K_{H_{2} O}}\left(q_{O_{2}}^{i n}-K_{H_{2} O} p_{H_{2} O}+2 K_{r} I\right) \\
\dot{T}_{s}= & \frac{1}{m_{s} C_{p s}}\left[\sum q_{i}^{\text {in }} \int_{T_{r e f}}^{T_{i n}} C_{p, i}(T) d T\right. \\
& \left.-\sum q_{i}^{\text {out }} \int_{T_{r e f}}^{T_{s}} C_{p, i}(T) d T-2 K_{r} I \Delta \hat{H}_{r}^{o}-V_{s} I\right] \\
V_{s}= & N_{0} \Delta E-r_{0} \exp \left[\alpha\left(\frac{1}{T_{s}}-\frac{1}{T_{0}}\right)\right] I \\
\Delta E= & {\left[\Delta E_{0}+\frac{R T}{2 F} \ln \left(\frac{p_{H_{2}} p_{O_{2}}^{(0.5)}}{p_{H_{2} O}}\right)\right] }
\end{aligned}
$$

For the component mass balances, $p_{i}$ is the partial pressure of component $i$, $T_{s}$ is the stack temperature, $\tau_{i}$ is the time constant for component $i$, described by $\frac{V}{K_{i} R T^{*}}$ with $V$ being the volume component $i$ is contained in, $K_{i}$ is the valve molar constant for component $i, R$ is the gas constant, $\tau_{i}^{*}=\left.\tau_{i}\right|_{T_{s}=T^{*}}$, and $T^{*}$ is the operating temperature. $q_{i}^{i n}, p_{i}$ are the inlet flow rate and partial pressure of component $i$, respectively, $K_{r}=\frac{N_{O}}{4 F}$, where $N_{O}$ is the number of cells in the stack and $F$ is Faraday's constant. Lastly $I$ is the load current. As for the energy balance, $m_{s}$ and $C_{p s}$ are the mass and heat capacity of the stack, $T_{r e f}$ and $T_{0}$ are the reference and feed temperatures, $C_{p, i}$ is the heat capacity of component $i, q_{i}^{\text {out }}$ is the outlet flow rate of gas $i, \Delta \hat{H}_{r}^{o}$ is the specific heat of reaction, $V_{s}$ is the stack voltage, $r_{0}$ is the internal resistance, $\alpha$ is the resistance slope, and $\Delta E_{0}$ is the standard cell potential.

\subsection{Control problem formulation with fault modeling}

The SOFC system modeled in Eq1 has three potential manipulated variables. Previous work has exploited this fact to implement multiple control configuration in the case of actuator failure (see [17]). However, here the focus 
will be on the accommodation of faults through model manipulation, and the main control configuration will be the one utilizing the hydrogen gas flow rate as the manipulated variable (this should be assumed unless otherwise noted). A similar approach can be used if other manipulated variables are chosen instead (the oxygen flow rate or the feed temperature).

The problem formulation is the same as in [17], where the controller design is based on the linearized system model about the desired operating set-point, where the time evolution of the system is given by:

$$
\begin{aligned}
& \dot{x}=A x+B \theta u \\
& \dot{\widehat{x}}=\widehat{A} \widehat{x}+\widehat{B} \widehat{\theta} u
\end{aligned}
$$

$$
u=K \widehat{x}
$$

where $x=\left[p_{H_{2}}^{\prime} p_{\mathrm{O}_{2}}^{\prime} p_{H_{2} \mathrm{O}}^{\prime} T_{s}^{\prime}\right]^{T}$ is the state vector of deviation variables from the desired steady state operation point $\left(x_{s s}=\left[\begin{array}{llll}0.3973 & 0.3142 & 0.2951 & 1162.8\end{array}\right]^{T}\right)$, $\widehat{x}$ is the model state which provides an estimate of $x, \widehat{A}, \widehat{B}$ are constant model matrices that approximate the linearized system matrices, $A$, and $B$ allowing for consideration of plant-model mismatch (see Eq 5 for the values of the nominal system state matrices). The effect of faults is captured through $\theta$ which, in general, is a diagonal matrix whose diagonal elements represent the fault or health status of each control actuator, where a value of 1 represents a healthy actuator and a value of zero reflects total failure. $\widehat{\theta}$ is an estimated fault parameter used in the control model and will serve as a key fault accommodation parameter. Finally, $u$ denotes the control action, with $K$ being the feedback controller gain.

Remark 1. It should be noted that the dimension of the $\theta, \widehat{\theta}$ matrices are dependent on the number of manipulated inputs, and that in this study they are both taken as scalars. 


$$
\begin{aligned}
A & =\left[\begin{array}{cccc}
-0.034998 & 0 & 0 & 0 \\
0 & -0.3139 & 0 & 0 \\
0 & 0 & -0.011666 & 0 \\
-8.9278 & -28.673 & -3.2569 & -0.010988
\end{array}\right], \\
B & =\left[\begin{array}{c}
0.0034721 \\
0 \\
0 \\
0.63486
\end{array}\right], \quad K=\left[\begin{array}{llll}
-12.4 & 0 & 0 & 0.047
\end{array}\right]
\end{aligned}
$$

Remark 2. Note that in general $\widehat{A} \neq A, \widehat{B} \neq B$, etc. In the case of this study a perfect model of the plant was assumed such that $\widehat{A}=A, \widehat{B}=B$, etc.

To analyze the stability and performance properties of the model-based controller of Eqs,24, we define an augmented state vector of the form $\xi=\left[\begin{array}{ll}x & e\end{array}\right]^{T}$, where $e=x-\widehat{x}$ is the model estimation error. The sampled-data closed-loop system can then be cast in the following form:

$$
\begin{aligned}
\dot{\xi} & =\Lambda \xi \\
e\left(t_{j}\right) & =0, \quad j \in\{0,1,2, \cdots\}
\end{aligned}
$$

where

$$
\Lambda=\left[\begin{array}{cc}
A+B \theta K & -B \theta K \\
(A-\widehat{A})+(B \theta-\widehat{B} \widehat{\theta}) K & \widehat{A}-(B \theta-\widehat{B} \widehat{\theta}) K
\end{array}\right]
$$

Note that the model estimation error is reset to zero at each sampling time due to the update of the model state using the actual sampled state. It can then be shown that the solution to the augmented system takes the following form:

$$
\xi(t)=e^{\Lambda\left(t-t_{j}\right)}\left(I_{o} e^{\Lambda \Delta} I_{o}\right)^{j} \xi_{0}, \quad t \in\left[t_{j}, t_{j+1}\right)
$$


where $\Delta=t_{j+1}-t_{j}$ is the sampling period and $I_{o}=\left[\begin{array}{cc}I & O \\ O & O\end{array}\right]$, with $I$ being the identity matrix. Due to the discrete sampled nature of the system it can be shown that closed-loop stability is assured if the maximum eigenvalue magnitude of the matrix $M:=I_{o} e^{\Lambda \Delta} I_{o}$ is less than 1, i.e., $\lambda_{\max }(M)<1$.

Remark 3. Note that in this study the linear controller was applied to the linearized model of the system. When implemented on the nonlinear system, the stability results are expected to hold locally (i.e., over a sufficiently small operating region). To expand the operating region of the controller, one may consider a linear time-varying model, where the state and input matrices $A, B, \widehat{A}, \widehat{B}$, are time-varying to allow for operation in multiple modes across multiple linearization regimes. Another approach is to use nonlinear control techniques to design the controller directly on the basis of the nonlinear system. In these approaches, however, the stability analysis of the sampled-data closed-loop system tends to be conservative in general, which typically hinders the ability to obtain an exact characterization of the closed-loop stability region.

Owing to the parametrization of the stability test matrix $M$ in terms of the various model, fault and control system parameters, the stability condition can be used to systematically explore the balance between these various parameters in influencing closed-loop stability. For example, for a fixed model and controller design parameters, the stability condition can be used to determine the range of tolerable fault magnitudes that do not compromise closed-loop stability. Alternatively, for a given fault size, the same condition can be used to identify the possible ranges for model and/or controller parameters that would ensure stability in the presence of the fault. This link is the basis for stability-based fault accommodation strategies.

\section{Fault detection and estimation via moving horizon optimization}

The first step in fault handling is the detection and estimation of faults. This is done through a moving-horizon optimization problem formulation (inspired 
by the formulation presented in [19]) in which an estimated value of $\theta$ (this will be referred to as $\theta^{*}$ ) is computed at each sampling time by minimizing the error between the sampled plant state and the estimated value provided by the fault diagnoser model. The cost function to be minimized is given by:

$$
J\left(\zeta_{j}, \bar{\theta}\right)=\sum_{p=j-N_{i}}^{j-1}\left(\left\|x[p+1]-\bar{x}\left(\zeta_{j}, \bar{\theta}\right)[p+1]\right\|^{2}\right)
$$

where

$$
\begin{aligned}
\zeta_{j} & =(x[j-q], u[j-q], \widehat{\theta}[j-q], \Delta[j-q]), \\
q & \in\left\{0,1,2, \ldots, N_{I}\right\}
\end{aligned}
$$

and $N_{I}$ is the size of the horizon window, where each point in $N_{I}$ is a time at which the plant state is sampled. $\bar{x}$ is obtained through initializing an embedded model of the system at $\bar{x}[i]=x[i]$ and then simulating the plant response over the sampling period using the $\zeta_{j}$ parameters fed to $J$ at which point $\bar{x}[p+1]$ is compared with $x[p+1]$, and then $\bar{x}$ is initialized at time $[p+1]$ and the process is repeated over the horizon window. By minimizing the cost function in Eq9 the value for the estimated fault parameter can be obtained at each time step $t_{j} \forall j \geq N_{I}$ as:

$$
\theta^{*}[j]=\underset{\bar{\theta}}{\operatorname{argmin}}\left\{J\left(\zeta_{j}, \bar{\theta}\right)\right\}
$$

Remark 4. It should be noted that the results of this work can be extended and generalized to the case when only output measurements are available at the sampling times. Specifically, in this case, a suitable state observer would need to be included in the control system to provide estimates of the system states from the available output measurements. These estimates can then be used to update the states of the controller and diagnoser models at the sampling times (e.g., see [20] for a similar approach used in the context of networked output feedback control). Fault estimation can be done by minimizing the difference 
(over the chosen horizon) between the measured output of the system available at the sampling times and the output of the diagnoser model embedded in the sensor (which depends on the tunable fault parameter). This leads to a cost function of the following form:

$$
J\left(\zeta_{j}, \bar{\theta}\right)=\sum_{p=j-N_{i}}^{j-1}\left(\left\|y[p+1]-\bar{y}\left(\zeta_{j}, \bar{\theta}\right)[p+1]\right\|^{2}\right)
$$

where $\zeta_{j}$ would no longer contain the exact states at the sampling times but the estimates generated by the state observer.

Unlike residual-based approaches (e.g., 17]) which allow only for fault detection, the above optimization-based approach provides capabilities for simultaneous detection, isolation and estimation of multiple faults, which is important for the selection of the appropriate fault accommodation strategy. The methodology in this section is also applicable to faults in measurement sensors or the plant itself through placement of a fault parameter matrix similar to that of the actuator fault matrices in this study. These types of faults can be modeled using the following general forms: $y=\theta C x$ for measurement sensor faults and $\dot{x}=A \theta x+B K x$ for plant faults. The fault estimator would then undergo the same logic of minimizing a suitable cost function. In the case of plant faults, however, $\theta$ would not necessarily be a diagonal matrix.

\section{Fault accommodation strategies}

\subsection{Performance metric characterization}

In order to accommodate a fault from a performance standpoint, a performance metric must be in place. The advantages of performance-based accommodation are that through the use of a performance metric, different stabilizing configurations can be compared objectively to decide which one is better suited to the needs of the process. Due to the sampled nature of the control system considered here, the extended $H_{2}$-norm is chosen as a suitable performance metric (e.g., see [21, 15]). The extended $H_{2}$-norm is used for measuring the 2-norm 
of a periodically sampled-data systems where continuous measurements are not available. It is a measure of the settling time of the performance output of a system after an impulse disturbance is introduced in a prescribed input.

To characterize the extended $\mathrm{H}_{2}$-norm as a function of the plant, model, fault and control system parameters, we reconsider the state-space representation in Eq2 but now with disturbances and a performance output introduced as follows:

$$
\begin{aligned}
\dot{x}(t) & =A x(t)+B \theta u(t)+E d(t) \\
z(t) & =M x(t)
\end{aligned}
$$

where $d$ is the disturbance input and $z$ is the performance output. In the case of the SOFC plant, the performance output chosen is the power output of the system. After some manipulations, the sampled-data closed-loop system can be formulated as:

$$
\begin{aligned}
\dot{\xi}(t) & =\Lambda \xi(t)+H d(t), t \in\left[t_{j}, t_{j+1}\right) \\
\xi\left(t_{j}\right) & =\left[\begin{array}{ll}
x^{T}\left(t_{j}\right) & 0
\end{array}\right]^{T}, j \in\{0,1,2,3, \ldots\} \\
z(t) & =N \xi(t)
\end{aligned}
$$

with $H=\left[\begin{array}{ll}E^{T} & E^{T}\end{array}\right]^{T}$ and $N=\left[\begin{array}{ll}M & O\end{array}\right]$. By solving Eq[13, it can be verified that the closed-loop response of the performance output to an impulse disturbance $d=\delta\left(t-t_{0}\right)$ can be expressed as:

$$
z(t)=N e^{\Lambda\left(t-t_{j}\right)}\left(I_{o} e^{\Lambda \Delta}\right)^{j} H, t \in\left[t_{j}, t_{j+1}\right)
$$

and the extended $\mathrm{H}_{2}$-norm can be computed as follows:

$$
\|G\|_{H_{2}}=\operatorname{trace}\left(H^{T} X H\right)^{1 / 2}
$$

where $X$ is the solution of the following discrete Lyapunov equation:

$$
M(\Delta)^{T} X M(\Delta)-X+W_{0}(0, \Delta)=0
$$


with $M(\Delta)=I_{o} e^{\Lambda \Delta}$ and $W_{o}(0, \Delta)=\int_{0}^{\Delta} e^{\Lambda^{T} t} N^{T} N e^{\Lambda t} d t$.

The extended $\mathrm{H}_{2}$-norm is of particular interest here seeing that it can be expressed in closed form and is also a function of the same parameters used to characterize closed-loop stability, namely $\theta, \widehat{\theta}, \Delta$, and $K$. This allows a quantitative assessment of the potential balance that exists between these various parameters in influencing closed-loop performance. For example, this characterization of the performance metric can be used to assess the extent of performance deterioration resulting from different fault situations. Similarly, for a given fault scenario, the impact of different choices of the control and model parameters on performance can be analyzed. Standard optimization techniques can be used, for example, to find the minimum extended $H_{2}$-norm as a function of these variables. This is useful in determining the best plan of action for the accommodation of faults.

\subsection{Performance-based fault accommodation}

Once a fault in need of accommodation has been deemed to occur, the controller must take a suitable corrective action to compensate for the effect of the fault. This is achieved by minimizing the performance objective (over one or more possible fault accommodation parameters), whilst ensuring closed-loop stability of the system. Note here that the performance objective is minimized because it denotes the settling time of the system from an impulse disturbance. For example, if a fault is established at time $t_{j}$ and $\widehat{\theta}$ is chosen as the fault accommodation parameter, the value that $\widehat{\theta}\left(t_{j+1}\right)$ should be assigned is determined by solving the following constrained optimization problem:

$$
\begin{aligned}
\widehat{\theta}\left(t_{j+1}\right) & =\min _{\widehat{\theta}}\left\{\|G\|_{H_{2}}\right\} \\
& \text { s.t. } \quad \lambda_{\max }\left(M\left(\bar{A}, \bar{B}, \widehat{A}, \widehat{B}, K, \theta^{*}, \widehat{\theta}, \Delta\right)\right)<1
\end{aligned}
$$

where $\bar{A}$ and $\bar{B}$ are the modeled values of $A$ and $B$ in the embedded fault estimator, and the constraint on the maximum eigenvalue magnitude is made to ensure closed-loop stability (see Section 2.2). In some cases, it may be necessary 
to perform the minimization of the extended $H_{2}$-norm over a constrained range of possible $\widehat{\theta}$ values to avoid unreasonably large values that may result from the solution of the unconstrained problem.

Remark 5. An accommodation strategy similar to the one given in Eq 17 can be used if other possible fault accommodation parameters are chosen (e.g., the feedback gain $K$, or the sampling period, $\Delta$ ). One can also optimize the performance index over multiple fault accommodation parameters simultaneously; this, however, comes at the expense of additional computational complexity, and the stability of the accommodated system needs to remain ensured.

\subsection{Implementation issues}

An important consideration in the implementation of fault-tolerant control is the need to minimize false detection alarms which may trigger unnecessary fault accommodation events that, at best, could lead to increased computational cost, and, at worst, could compromise performance and lead to instability. This is important, especially in the presence of unmodeled uncertainties and unknown perturbations that could impact the optimization-based fault estimation results. Determining weather or not a fault is in need of accommodation, therefore, requires careful consideration. For example, once a change in the estimated fault parameter has been observed, the controller must determine if the fault is new or one that has had previous accommodation. This determination can be made through the use of a reference fault parameter, $\theta_{\text {ref }}^{*}$, which is updated every time a fault is accommodated. An example heuristic that one can employ here is to check if the mean of the estimated fault parameter over the past $N_{\theta^{*}}$ sampled data points, $\mu$, has deviated by some pre-defined threshold, $\theta_{\text {thresh }}^{*}$, i.e.,

$$
\begin{gathered}
\left|\theta_{\text {ref }}^{*}-\mu\right|>\theta_{\text {thresh }}^{*} \\
\mu=\operatorname{mean}\left\{\theta^{*}\left[p-N_{\theta^{*}}\right], \theta^{*}\left[p-N_{\theta^{*}}+1\right], \cdots, \theta^{*}[p]\right\}
\end{gathered}
$$


If such a deviation is observed, then the controller suspects that a new fault has occurred.

Another important aspect is the need to provide some robustness margin against unmodeled disturbances. Given that the performance of the system under impulse-like disturbances is considered (which is captured by the extended $H_{2}$-norm), it is important that such unmodeled disturbances not be mistaken for faults and thus not trigger unnecessary fault accommodation measures. One approach to deal with this is to exploit the fact that any impulse-like disturbance on the system will rapidly shift the states, and will be met by the embedded model attempting to find an estimated value of the fault size that would cause this stark shift, thus creating a rapid change in the estimated fault parameter. With this knowledge, a secondary condition for the determination of possible faults would be to require that the estimated fault parameter not exceed some pre-defined threshold, $\theta_{\max }^{*}$, that would not be expected to be reached under normal operating conditions. In other words, if the following condition holds:

$$
\max \left\{\left|\theta^{*}\left[p-N_{\theta^{*}}\right]\right|,\left|\theta^{*}\left[p-N_{\theta^{*}}+1\right]\right|, \ldots,\left|\theta^{*}[p]\right|\right\}<\theta_{\max }^{*}
$$

as well as that in Eqs 18] 19, then the controller decides that a fault has occurred that must be accommodated.

Since the fault accommodation strategy is based on the estimated fault size, it is important once a fault has been suspected to wait for the estimated fault value to settle prior to taking corrective action. This would have the benefit of not taking unnecessary action during disturbances, as well as reducing the computational load of calculating the accommodation action every time $\theta^{*}$ changes (recall that fault accommodation is based on solving the constrained optimization problem in Eq(17). The settling time of the estimated fault parameter is dependent on the size of the horizon used in the fault estimation scheme. The estimated fault value gradually changes as additional faulty data points are incorporated into the data set used for estimation. Eventually the estimated value settles at a constant once all data points in the horizon are ones containing the 
fault. An example heuristic that can be used here is to trigger an optimization of the control action only when the deviations in successive estimates of $\theta^{*}$ fall below a certain threshold, $\epsilon_{\theta^{*}}$, i.e.,

$$
\begin{gathered}
\left|\mu^{\prime}-\theta^{*}[p]\right|<\epsilon_{\theta^{*}} \\
\mu^{\prime}=\operatorname{mean}\left\{\theta^{*}\left[p-N_{\theta^{*}}\right], \theta^{*}\left[p-N_{\theta^{*}}+1\right], \cdots, \theta^{*}[p-1]\right\}
\end{gathered}
$$

where $\epsilon_{\theta^{*}}$ is a tunable parameter that $\theta^{*}$ must remain within for the optimizationbased fault accommodation to take place. A summary of the fault detection, estimation and accommodation framework is given in Fig 1.

\section{Simulation study}

The objective of this section is to illustrate the implementation of the developed fault estimation and accommodation framework to the SOFC example introduced earlier in Section 2.

\subsection{Performance gains through use of fault accommodation}

In prior work ([17]), stability regions were shown to aid in understanding the impact uncertainty had on closed-loop stability of the SOFC plant and used to develop a stability-based control system reconfiguration strategy that maintains stability in the presence of controller failures. In this study, a different approach is taken, and that is to show explicitly the dependence of stability and performance on the actual and modeled faults in the system. To this end, Fig 2 is a contour plot of $\lambda_{\max }(M)$ as a function of $\theta$ and $\widehat{\theta}$. The uncolored region is the region enclosed by the unit contour line and is therefore the stable region of operation. It can be seen from this plot that for any given fault, where $\theta \gtrsim 7.44$ and $\widehat{\theta}=1$, the closed-loop system will be unstable. Furthermore, there is a wide range of possible accommodation scenarios that can be implemented through altering $\widehat{\theta}$ that lead to stable post-accommodation operation. 


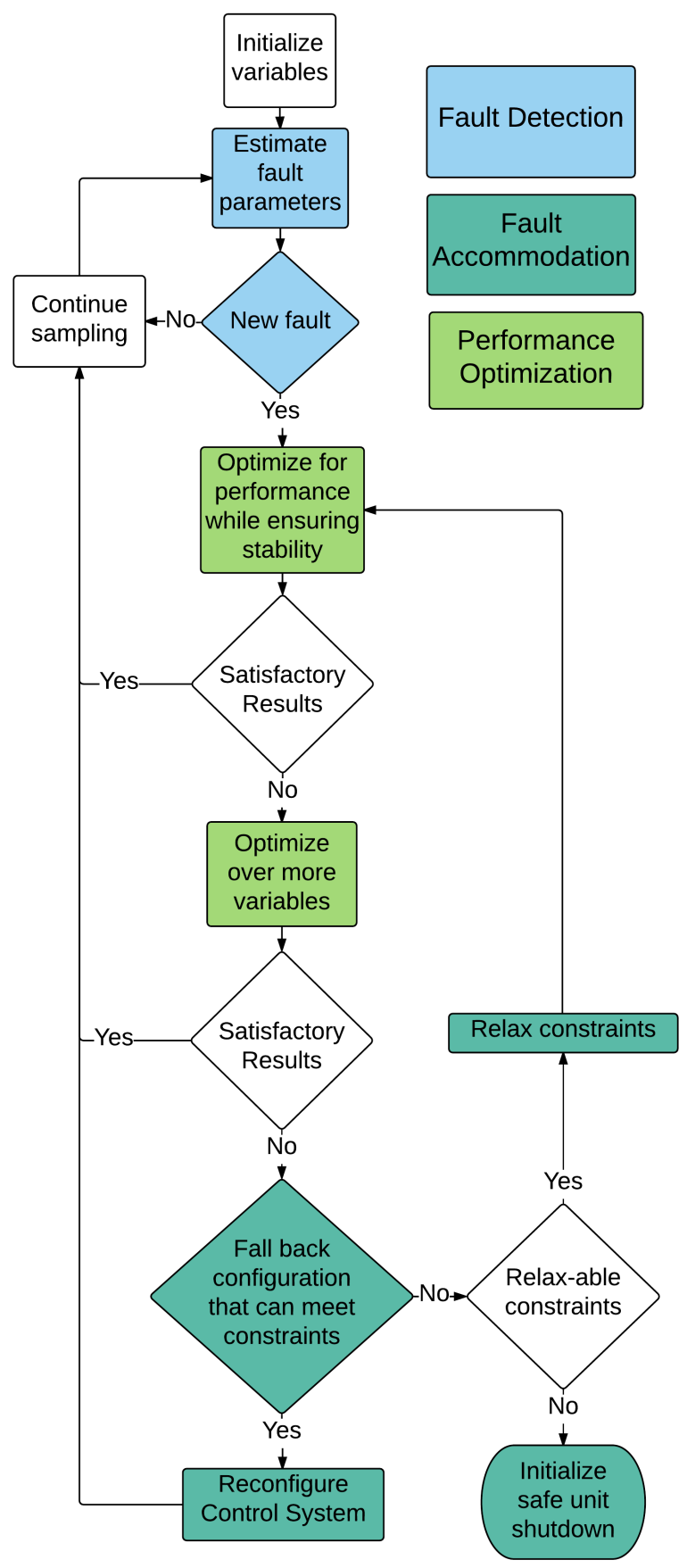

Figure 1: A flowchart of the integrated fault estimation and accommodation methodology. 
Fig[3]shows the advantage of fault accommodation when a destabilizing fault of $\theta=7.9$ occurs at $t=500 \mathrm{~s}$. The closed-loop system with the accommodated fault $(\widehat{\theta}=15)$ maintains a steady power output trajectory (black profile) in the face of a fault in the actuator manipulating the hydrogen flow rate, whereas the power output of the closed-loop system with no fault accommodation $(\widehat{\theta}=1)$ is shown to be unstable for the same fault (red profile).

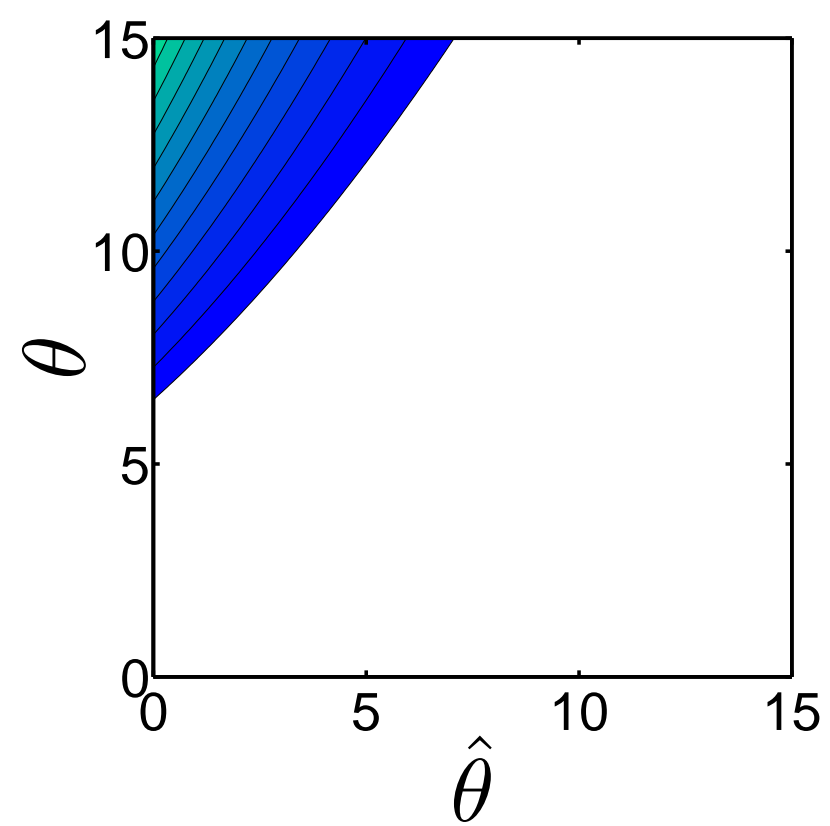

Figure 2: Characterization of the sampled-data controlled SOFC stability region: A contour plot of $\lambda_{\max }(\theta, \widehat{\theta})$.

343 Given the wide range of values of $\widehat{\theta}$ that can be used for stability-based 344 accommodation, an optimal post-fault choice of the value of $\widehat{\theta}$ would naturally 345 be desirable, and this is where the performance criteria is applied. It is sought 346 to find the accommodation strategy for the model fault parameter that gives 347 the best performance while maintaining closed-loop stability. A contour plot of 348 the extended $H_{2}$-norm as a function of $\theta$ and $\widehat{\theta}$ is shown in Fig, where the extended $\mathrm{H}_{2}$-norm is the contour value on this plot and the smaller this norm 


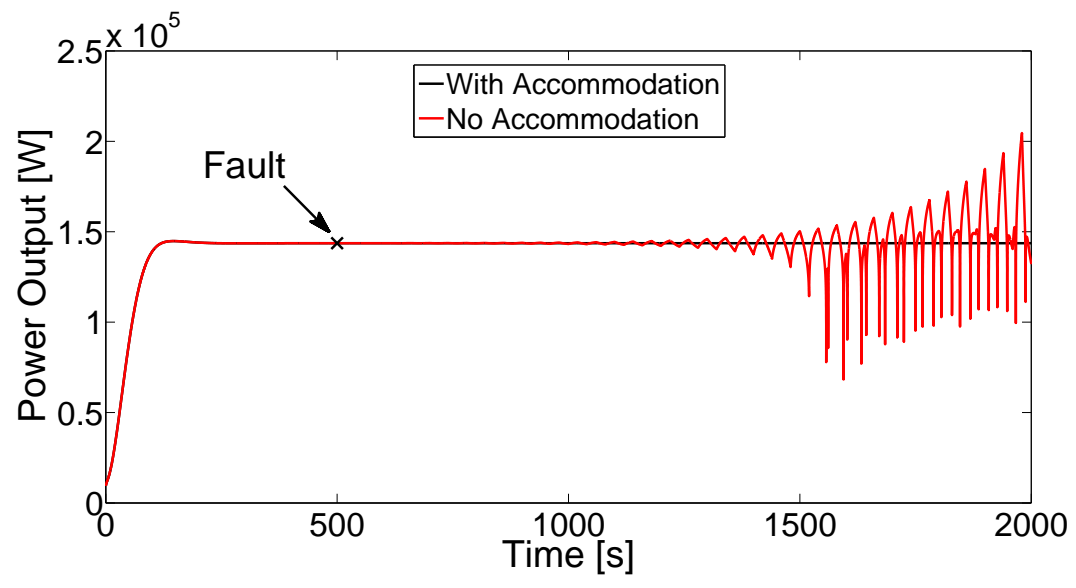

Figure 3: Evolution of the power output in the presence of a destabilizing fault.

the better the system performs (i.e., the faster the recovery from disturbances). It can be seen that, for a given value of $\widehat{\theta}$, as $\theta$ increases beyond 1 (i.e., the fault severity increases), extended $H_{2}$-norm increases and thus the performance deteriorates.

Note that the performance criterion is only meaningful in the region where the closed-loop system is stable (see Fig 2). Due to the shape of the stability contour plot, simply setting $\widehat{\theta}=\theta^{*}$ can be seen to place the system in a stable region of operation. This is referred to as "minimal accommodation" in the sense that the controller needs no knowledge of the stability or performance of the system as a function of $\widehat{\theta}$. This minimal accommodation strategy assumes that if the model matches the plant, then this will be a suitable accommodation strategy. It can be seen with the aid of the performance contour plot of the extended $\mathrm{H}_{2}$-norm (Fig 4), however, that there is in fact a better solution to the accommodation problem from a performance standpoint than simply setting $\widehat{\theta}=\theta^{*}$. It should be noted that the shape of this particular contour plot is unique to the chosen control configuration, and that, in general, setting $\widehat{\theta}=\theta^{*}$ cannot be guaranteed to result in a stable post-fault system even if $\theta^{*}=\theta$.

To illustrate the performance-based accommodation, we consider a non- 


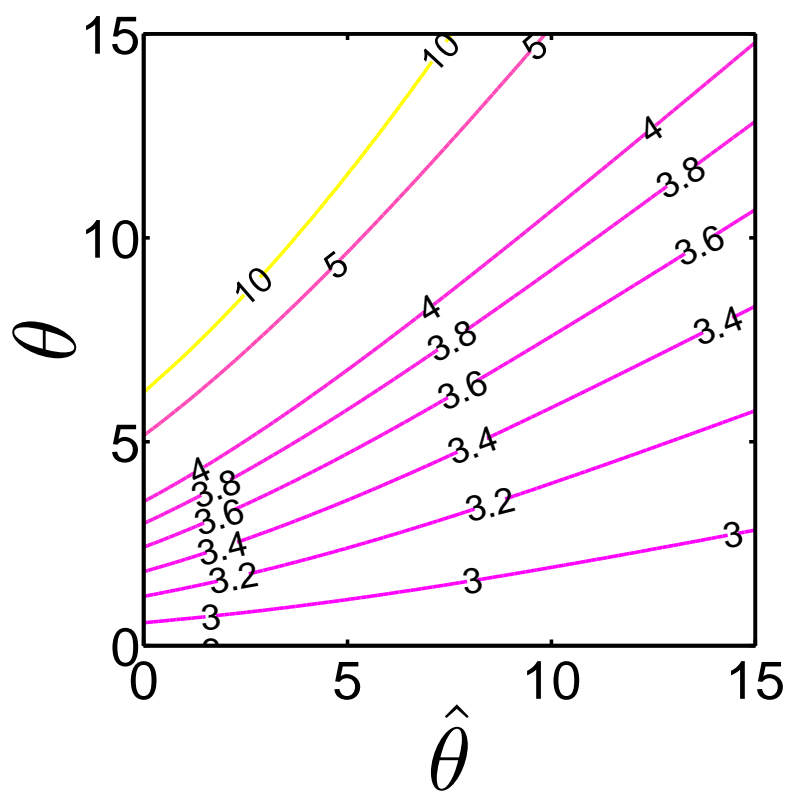

Figure 4: Characterization of the performance levels of the SOFC plant: A contour plot of the extended $\mathrm{H}_{2}$-norm as a function of $\theta$ and $\widehat{\theta}$ ). 
destabilizing fault in the control actuator at $t=1000 \mathrm{~s}$, as shown in Fig 5 (red profile). This figure also shows the evolution of the estimated fault parameter (blue profile) which is computed from Eq11. It can be seen that the value of $\theta^{*}$ steps its way closer to the actual value of the fault parameter every sampling period $(\Delta=20 s)$. Once $\theta^{*}$ has settled in accordance with Eqs, 18, 19, Eq, 20, and Eqs 21, 22, then fault accommodation is triggered and $\widehat{\theta}$ is adjusted per Eq17 (green profile) to minimize performance losses while maintaining closedloop stability. The impact of this fault accommodation strategy on closed-loop performance is shown in Fig 6 and Fig 7.

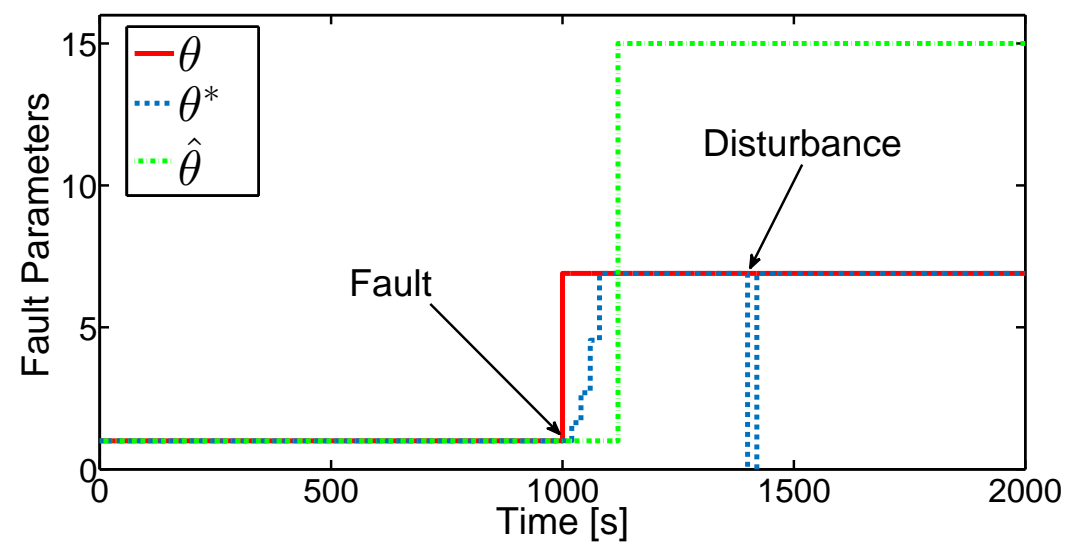

Figure 5: Fault parameter estimation for a performance-degrading fault.

Fig[6 shows the accommodation result with $\widehat{\theta}$ being optimized to minimize the extended $\mathrm{H}_{2}$-norm. This shows the advantage of accommodating a performance-degrading fault, $\theta=6.9$, occurring at $t=1000$ followed by a disturbance of $10 \%$ of the steady state value of the $\mathrm{O}_{2}$ flow rate. This figure shows the accommodated power output response in black (extended $H_{2}$-norm $=3.23$ ) and the response with no accommodation in red (extended $H_{2}$-norm $=7.42$ ). The minimal accommodation strategy, where $\widehat{\theta}=\theta^{*}$ is not shown in the plot, but yields an extended $\mathrm{H}_{2}$-norm of 3.78 . It can be seen that the system with fault accommodation has a much smoother and faster response back to the steady state post the fault and disturbance events. 
One can also see in Fig 5 that the disturbances occurring at $t=1400 \mathrm{~s}$ do not trigger any change in $\widehat{\theta}$ even though $\theta^{*}$ changes drastically. This is due to the precautions put in place from Eqs, 18,20. It should be noted again that these were obtained from the knowledge of the type of disturbances that would be affecting the system.

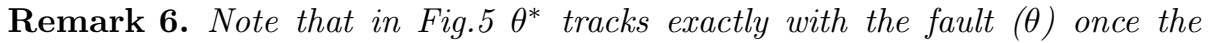
horizon contains all faulted data. This is due to the fact that no plant-model mismatch was considered, i.e., $\widehat{A}=A, \widehat{B}=B$, etc. When uncertainties are introduced in the context of plant-model mismatch, where $\widehat{A} \neq A, \widehat{B} \neq B$, then the resulting $\theta^{*}$ would be a range of values that the actual fault, $\theta$, would lie in, $\theta \in \theta^{*}$; the end result being an upper and lower bounds on the fault, $\min \left(\theta^{*}\right) \leq$ $\theta \leq \max \left(\theta^{*}\right)$, instead of the exact estimate, $\theta^{*}=\theta$, obtained in the case with no uncertainty. Note that the accommodation parameter $\widehat{\theta}$ must be stabilizing for all potential values of the fault parameter, $\theta \in \theta^{*}$.

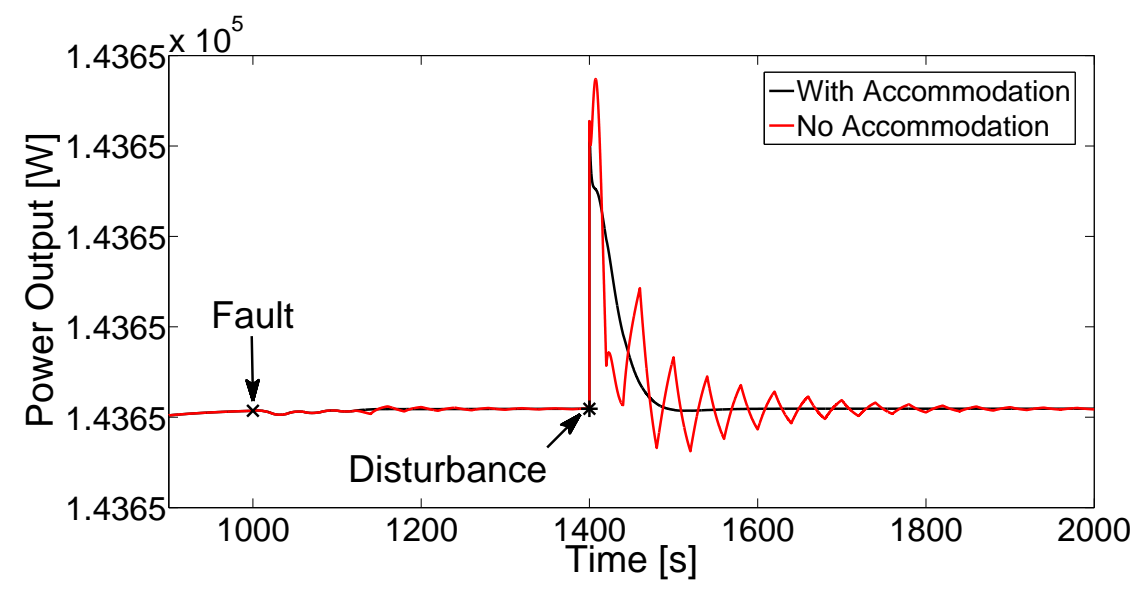

Figure 6: Evolution of power output subject to a performance-degrading fault followed by an impulse disturbance in the oxygen feed flow rate.

Fig [7 shows a similar accommodation result as in Fig 6 but with a disturbance of $10 \%$ of the steady state value of the stack temperature introduced into 
the feed temperature following the fault event. This figure shows the accommodated power output response in black (extended $H_{2}$-norm $=0.012$ ) and the response with no fault accommodation in red (extended $H_{2}$-norm $=0.028$ ). The minimal accommodation strategy with $\widehat{\theta}=\theta^{*}$ is not shown in the plot, but has an extended $H_{2}$-norm of 0.014 . It can be seen that the system with accommodation has a much smoother and faster response back to the steady state value post the fault and disturbance events.

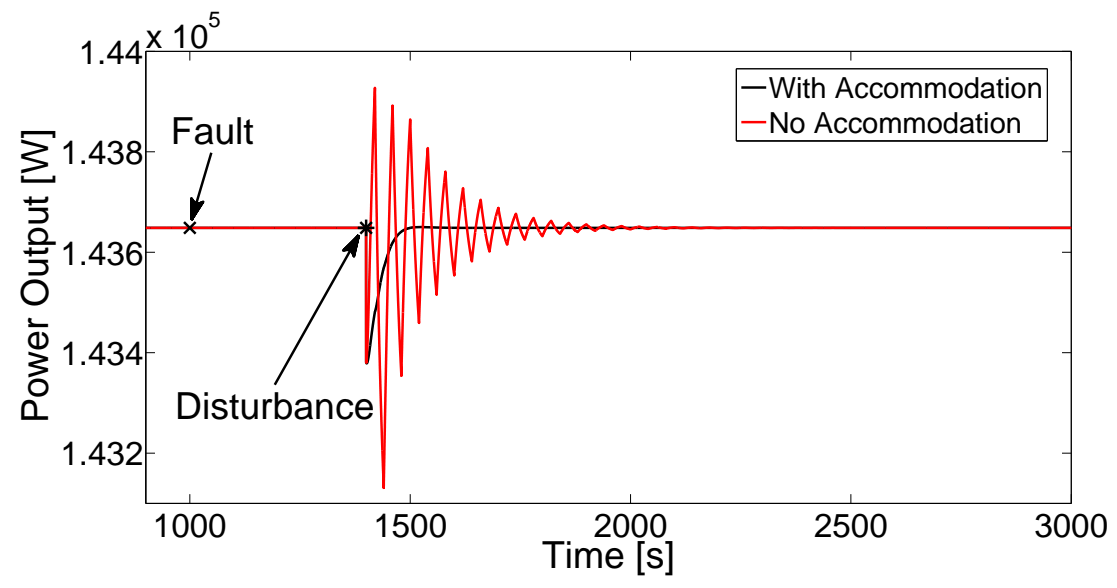

Figure 7: Evolution of power output subject to a performance-degrading fault followed by an impulse disturbance in the feed temperature.

\subsection{Choice of control configuration}

By examining the values of the extended $H_{2}$-norm obtained from the accommodation results reported in the previous subsection, it can be observed that the achievable values were far lower for the configuration where the manipulated variable was the hydrogen feed flow rate and the disturbance was introduced in the inlet feed temperature, as opposed to the configuration where the oxygen feed flow rate was used as the disturbance variable. It should also be noted that the ability of the control system to accommodate faults was far greater in the latter configuration. This can be seen in Fig 8 and Fig 9 which depict the dependence of the extended $H_{2}$-norm on the fault parameter for 
both configurations. It can be seen that faults in the control actuator have a far greater impact on closed-loop performance, when performance is measured with respect to recovery from disturbances in the oxygen feed flow rate, where as performance with respect to disturbances in the feed temperature is barely sensitive to changes in the fault parameter (over the stabilizing range). In Fig 8 for example, it can be seen that various values of $\theta$ can yield quite stark differences in the extended $\mathrm{H}_{2}$-norm magnitude. This is likely due to the underlying physical principles governing the system, where adjusting the hydrogen flow has a far greater impact on the power output when the oxygen flow rate is fluctuating than if the inlet feed temperature changes. However, this analysis does give insight into the importance of selecting proper control variables when knowledge of the disturbance variables is available. This analysis of the ability of the control system to accommodate faults could also be applied in more complex systems where the relationships between the available manipulated variables and expected disturbance variables are not as clear.

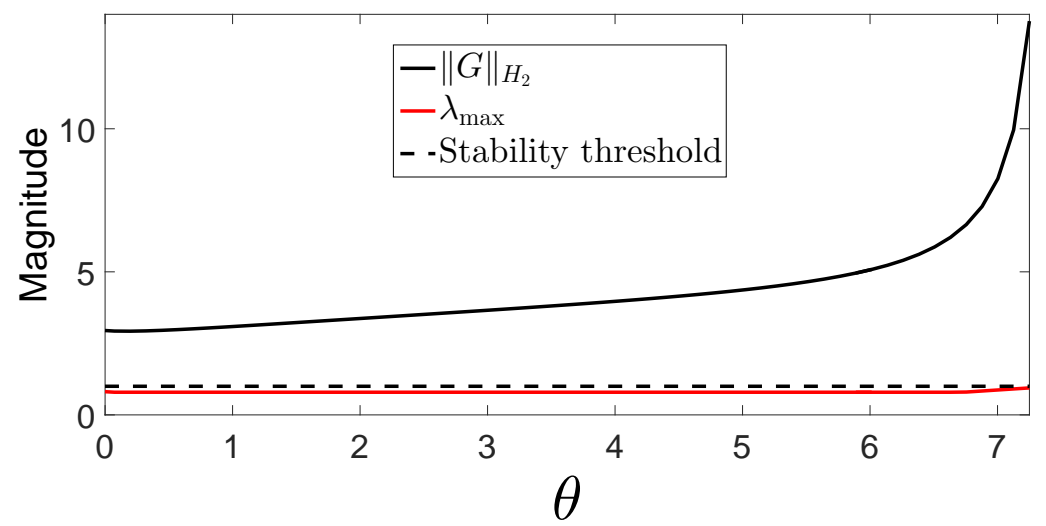

Figure 8: Magnitudes of the maximum eigenvalue of $M$ and the extended $H_{2}$-norm as functions of $\theta$ (with $\widehat{\theta}=1$ ) for the case when performance is measured with respect to disturbances in the oxygen feed flow rate.

Finally, Fig 10 and Fig 11 show the extent to which fault accommodation via adjusting $\widehat{\theta}$ can improve performance for both configurations. It can be 


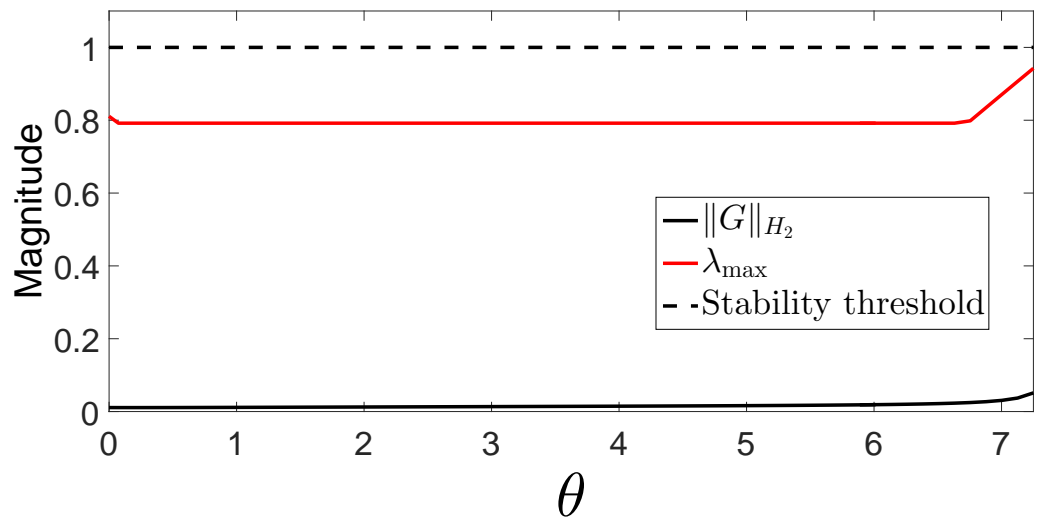

Figure 9: Magnitudes of the maximum eigenvalue of $M$ and the extended $H_{2}$-norm as functions of $\theta$ (with $\widehat{\theta}=1$ ) for the case when performance is measured with respect to disturbances in the feed temperature.

seen that the choice of $\widehat{\theta}$ can make a substantial impact on performance recovery when disturbances are considered in the oxygen feed flow rate, where as it has minimal impact on performance when the disturbance influences the feed temperature (note that the extended $H_{2}$-norm is only plotted over the range where $\left.\lambda_{\max }(M)<1\right)$. The latter configuration appears to be inherently robust from a performance standpoint to the faults tested here as can be seen from Fig 9 , however, the selection of $\widehat{\theta}$ for the fault shown in Fig 11 has little effect on performance, as can be seen in the extended $H_{2}$-norm values for the accommodation and no accommodation states pertaining to Fig:7.

\subsection{Benefits of control system reconfiguration}

So far, we have shown that fault accommodation measures, such as updating the model parameters, can be used to minimize performance losses following the occurrence of faults. In this subsection, we show that in some cases, reconfiguring the control system (e.g., by switching to an alternative fault-free manipulated input) can further improve closed-loop performance beyond what can be achieved with fault accommodation measures alone. To this end, we can 


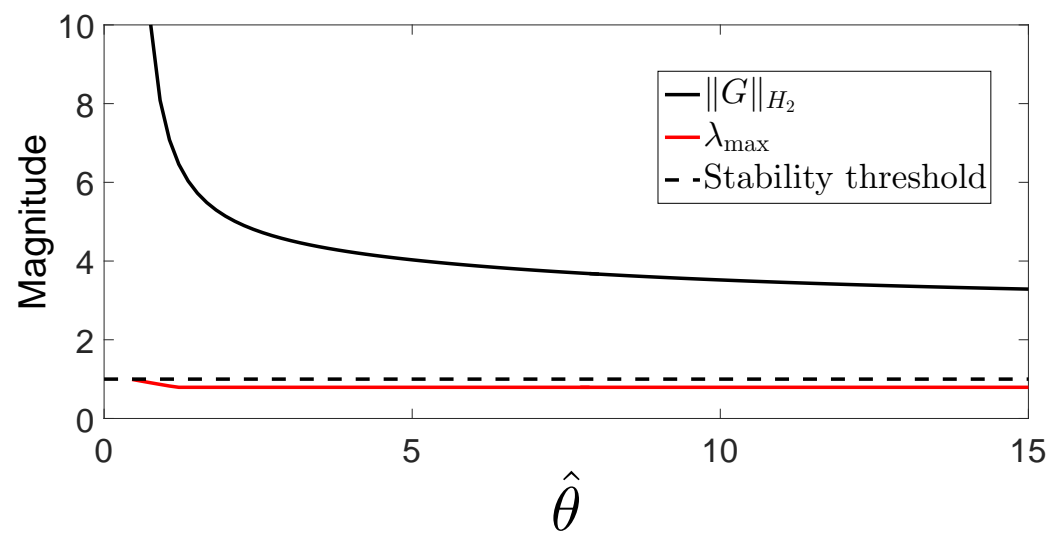

Figure 10: Magnitudes of the maximum eigenvalue of $M$ and the extended $H_{2}$-norm as functions of $\widehat{\theta}$ (with $\theta=6.9$ ) for the case when performance is measured with respect to disturbances in the oxygen feed flow.

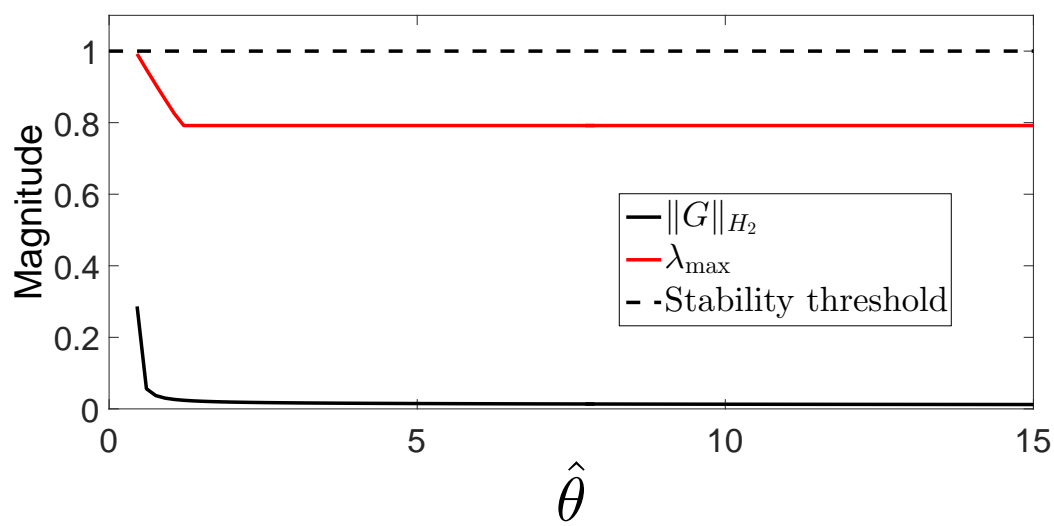

Figure 11: Magnitudes of the maximum eigenvalue of $M$ and the extended $H_{2}$-norm as functions of $\widehat{\theta}$ (with $\theta=6.9$ ) for the case when performance is measured with respect to disturbances in the feed temperature. 
see from Fig 10 that if the nominal system were to experience a fault such that $\theta=6.9$, then the best case scenario for recovery through fault accommodation (by adjusting $\widehat{\theta}$ ) would yield an extended $H_{2}$-norm value of $\approx 3.3$. However, if the control system were to switch to an alternative configuration in which the feed temperature, rather than the hydrogen flow rate, is used as the manipulated input, then as can be seen in Fig,12, an even smaller performance index (i.e., an improved performance) can be achieved for all values of $\widehat{\theta}$. The best performance is attained near $\widehat{\theta}=1$. This result suggests that in this case, and from a performance point of view, control system reconfiguration may be a better option than fault accommodation.

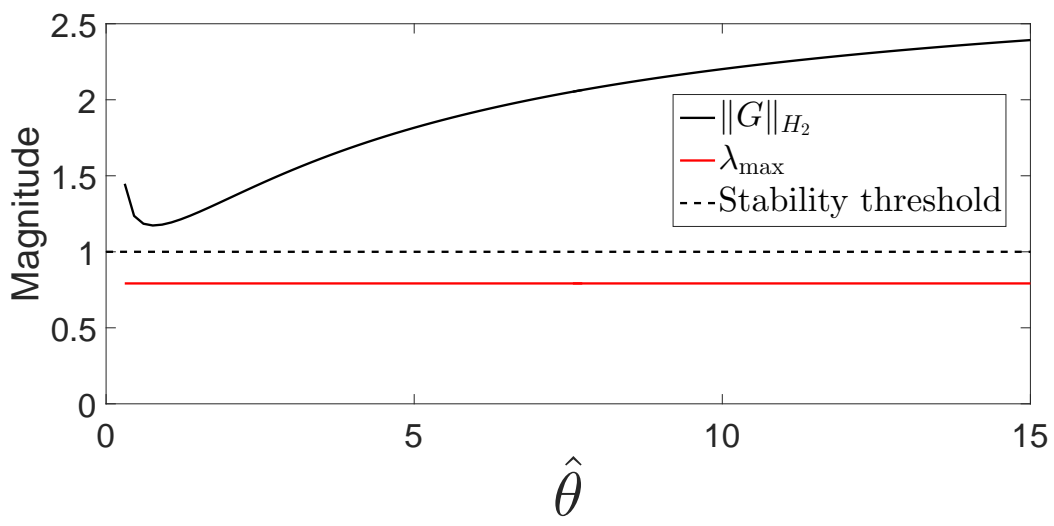

Figure 12: Magnitude of the maximum eigenvalue and the extended $\mathrm{H}_{2}$-norm as functions of $\widehat{\theta}$, with $\theta=1$, when the manipulated input is chosen as the feed temperature, and the disturbances occur in the oxygen feed flow.

In addition to performance gains through control system reconfiguration, it can be seen from Fig 13 that if the aforementioned reconfiguration strategy is implemented, then the newly reconfigured system would exhibit some degree of robustness to potential future faults in the new actuator configuration. Specifically, it can be seen that the extended $\mathrm{H}_{2}$-norm profile remains relatively flat (and well below 2) over a certain range of $\theta$ values around the healthy (faultfree) value of 1 , thus indicating minimal performance degradation for a certain 


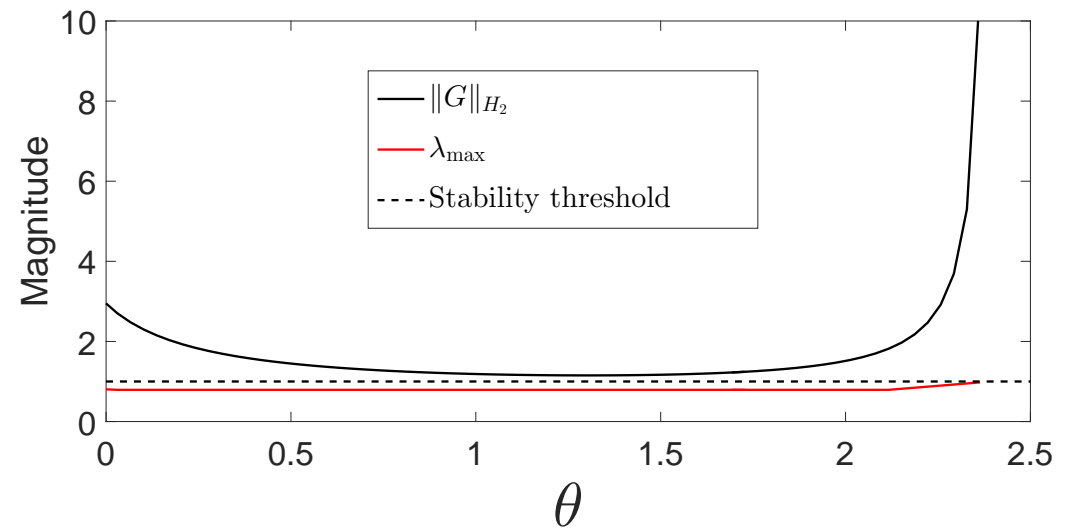

Figure 13: Magnitude of the maximum eigenvalue and the extended $H_{2}$-norm as functions of $\theta$, with $\widehat{\theta}=1$, when manipulated input is chosen as the feed temperature, and the disturbances occur in the oxygen feed flow.

range of faults. This can be compared to the result in Fig 8 for the range of values that $\theta$ can take and still have a reasonable extended $H_{2}$-norm value.

Finally, it should be noted that for Figs $8\left[13\right.$ the extended $H_{2}$-norm is only plotted for values of $\theta$ or $\widehat{\theta}$ (whichever is relevant) when $\lambda_{\max }(M)<1$, that is to say for values at which the closed-loop system in question is stable. This is due to the fact that there is no meaning in the performance index for an unstable system. This restriction is the cause for any discontinuities/asymptotes that may appear in the various plots.

\section{Concluding remarks}

In this work, a methodology for the design of local model-based fault-tolerant control systems for DERs was developed and illustrated using a simulated model of a SOFC system. The framework integrates model-based feedback control, data-based fault estimation and performance-based fault accommodation. Fault estimation was realized via a moving-horizon optimization scheme that used past data to obtain an estimate of the fault magnitude. The fault estimation 
scheme was complemented by a set of practical implementation guidelines aimed at minimizing false detection alarms. Based on a suitably chosen performance metric for the closed-loop system, a performance-based fault accommodation strategy was then devised to minimize fault-induced performance losses, while maintaining closed-loop stability.

When placed in the wider context of fault-tolerant control of DER networks, the focus of the current study has been on the detection and handling of faults solely at the local (i.e., the DER unit) level, without consideration of possible supervisory oversight or intervention. This emphasis is motivated by the fact that timely fault mitigation an protection measures at the local level are essential (when feasible) for preventing the effects of local faults from cascading through the system. However, in cases where local fault accommodation is no longer feasible, an intervention by a higher-level supervisor that takes into account the network-level dynamics becomes necessary to ensure fault-tolerance. The design of a supervisory fault-tolerant control system for DERs is the subject of ongoing research work.

\section{Acknowledgments}

Financial support by NSF, CBET-1438456, is gratefully acknowledged.

\section{References}

[1] K. Tomsovic, T. Hiyama, Intelligent control methods for systems with dispersed generation, in: Proceedings of IEEE Power Engineering Society Winter Meeting, Columbus, OH, 2001, pp. 913-917.

[2] F. Jurado, J. Saenz, Adaptive control of a fuel cell-microturbine hybrid power plant, in: Proceedings of IEEE Power Engineering Society Summer Meeting, Chicago, IL, 2002, pp. 76-81.

[3] M. Illindala, G. Venkataramanan, Control of distributed generation systems to mitigate load and line imbalances, in: Proceedings of IEEE Power Electronics Specialists Conference, Cairns, Australia, 2002, pp. 2013-2018. 
[4] M. Marwali, A. Keyhani, Control of distributed generation systems-part I: Voltages and currents control, IEEE Transactions on Power Electronics 19 (2004) 1541-1550.

[5] R. Roberts, J. Brouwer, F. Jabbari, T. Junker, H. Ghezel-Ayahg, Control design of an atmospheric solid oxide fuel cell/gas turbine hybrid systems: Variable versus fixed speed has turbine operation, Journal of Power Sources 161 (2006) 484-491.

[6] R. H. Lasseter, Microgrids and distributed generation, Journal of Energy Engineering 133 (2007) 144-149.

[7] K. Ro, S. Rahman, Control of grid-connected fuel cell plants for enhancement of power system stability, Renewable Energy 28 (2003) 397-407.

[8] A. Paradkar, A. Davari, A. Feliachi, T. Biswas, Integration of a fuel cell into the power system using an optimal controller based on disturbance accommodation control theory, Journal of Power Sources 128 (2004) 218230.

[9] K. Sedghisigarchi, A. Feliachi, Dynamic and transient analysis of power distribution systems with fuel cells-part II: control and stability enhancement, IEEE Transactions on Energy Conversion 19 (2004) 429-434.

[10] Z. Miao, M. A. Choudhry, R. L. Klein, L. Fan, Study of a fuel cell power plant in power distribution system - part II: Stability control, in: Proceedings of IEEE Power Engineering Society General Meeting, St. Paul, MN, 2004, pp. 2226-2231.

[11] M. I. Marei, E. F. El-Saadany, M. M. A. Salama, A novel control algorithm for the DG interface to mitigate power quality problems, IEEE Transactions on Power Delivery 19 (2004) 1384-1392.

[12] S. Barsali, M. Ceraolo, P. Pelacchi, D. Poli, Control techniques of dispersed generators to improve the continuity of electricity supply, in: Proceedings of IEEE Power Engineering Society Winter Meeting, 2002, pp. 789-794. 
[13] L. M. Tolbert, H. Qi, F. Z. Peng, Scalable multi-agent system for real-time electric power management, in: Proceedings of IEEE Power Engineering Society Summer Meeting, Vancouver, BC, Canada, 2001, pp. 1676-1679.

[14] A. L. Dimeas, N. D. Hatziargyriou, Operation of a multiagent system for microgrid control, IEEE Transactions on Power Systems 20 (2005) 14471455 .

[15] Y. Sun, S. Ghantasala, N. H. El-Farra, Networked control of distributed energy resources: Application to solid oxide fuel cells, Ind. Eng. \& Chem. Res. 48 (2009) 9590-9602.

[16] W. Qi, J. Liu, X. Chen, P. D. Christofides, Supervisory predictive control of standalone wind/solar energy generation systems, IEEE Trans. Contr. Syst. Tech. 19 (1) (2011) $199-207$.

[17] Y. Sun, S. Ghantasala, N. H. El-Farra, Monitoring and fault-tolerant control of distributed power generation: Application to solid oxide fuel cells, in: Proceedings of American Control Conference, 2010, pp. 448-453.

[18] A. M. Mursheda, B. Huang, K. Nandakumara, Control relevant modeling of planer solid oxide fuel cell system, Journal of Power Sources 163 (2007) 830-845.

[19] S. Samar, D. Gorinevsky, S. Boyd, Embedded estimation of fault parameters in an unmanned aerial vehicle, in: Proc. of 2006 IEEE International Conference on Control Applications, Munich, Germany, 2006, pp. 32653270.

[20] Y. Sun, N. H. El-Farra, A quasi-decentralized approach for networked state estimation and control of process systems, Ind. Eng. \& Chem. Res. 49 (2010) 7957-7971.

[21] L. A. Montestruque, P. J. Antsaklis, Performance evaluation for modelbased networked control systems, in: Netw. Emb. Sens. and Cntrl., LNCIS, 
Vol. 331, P.J. Antsaklis, P. Tabuada (Eds.), Springer-Verlag, Berlin, 2006,

pp. 231-249. 